\title{
Requirement of focal adhesion kinase in branching tubulogenesis Wei-Chun Wei ${ }^{1}$, Anna K Kopec ${ }^{2}$ and Ming-Jer Tang*1,3,4
}

\author{
Address: ${ }^{1}$ Institute of Basic Medical Sciences, National Cheng Kung University Medical College, Tainan 701, Taiwan, ${ }^{2}$ Department of Biochemistry \\ \& Molecular Biology, Michigan State University, East Lansing, MI 48824-1319 USA, ${ }^{3}$ Department of Physiology, National Cheng Kung University \\ Medical College, Tainan 701, Taiwan and ${ }^{4}$ Center of Gene Regulation and Signal Transduction, National Cheng Kung University Medical College, \\ Tainan 701, Taiwan \\ Email: Wei-Chun Wei - weichun.wei@gmail.com; Anna K Kopec - akopec@msu.edu; Ming-Jer Tang* - mjtang1@mail.ncku.edu.tw \\ * Corresponding author \\ Published: 12 January 2009 \\ Received: 17 October 2008 \\ Journal of Biomedical Science 2009, 16:5 doi:10.1186/1423-0127-16-5 \\ Accepted: 12 January 2009 \\ This article is available from: http://www.jbiomedsci.com/content//6/I/5 \\ (c) 2009 Wei et al; licensee BioMed Central Ltd. \\ This is an Open Access article distributed under the terms of the Creative Commons Attribution License (http://creativecommons.org/licenses/by/2.0), \\ which permits unrestricted use, distribution, and reproduction in any medium, provided the original work is properly cited.
}

\begin{abstract}
We previously demonstrated that $\alpha 3 \beta$ I integrins are essential to hepatocyte growth factor (HGF)independent branching tubulogenesis in Mardin-Darby Canine Kidney (MDCK) cells. However, the involvement of integrin downstream signaling molecules remains unclear. In the present study, we successfully isolated cell lines possessing different tubulogenic potentials from the MDCK cells; cyst clones (CA4, CA6) forming cystic structures when cultured in $0.3 \%$ type I collagen gel and mass clones (M6IO, M6II, M6I2) forming aggregated masses. Cyst clones maintained cystic structure in $0.1 \%$ collagen gel, whereas mass clones spontaneously developed into tubules. Both clones exhibited various morphologies when cultured on a dish: cyst clones formed aggregated islands, while mass clones were more scattered and exhibited higher migration capacity. Among several focal adhesion machinery proteins examined, only the expression and phosphorylation level of focal adhesion kinase (FAK) in mass clones was higher than in cyst clones, while other proteins showed no obvious differences. However, overexpression of wild type FAK in CA6 cells did not facilitate branching tubule formation in $0.1 \%$ collagen gel. Targeted decrease in the expression level of FAK in $\mathrm{M} 610$ cells with the application of antisense cDNA resulted in a marked reduction of branching tubule formation in $0.1 \%$ collagen gel and showed a down-regulation of fibronectin assembly, which is known to promote tubulogenesis. In contrast, overexpression of wild type FAK in CA6 cells had no effect on fibronectin assembly. Taken together, our data demonstrates that FAK is required, but not sufficient for HGF-independent branching tubulogenesis in MDCK cells.
\end{abstract}

\section{Background}

Epithelial branching tubulogenesis plays an essential role in the development of several tissues such as kidney, lung, salivary and mammary glands, and the study of the underlying mechanisms has been of both basic and clinical interest. Mardin-Darby canine kidney (MDCK) cells cultured in collagen gel are widely used as the simplest and most convenient model for studying tubulogenesis. Clone II 3B5, isolated from MDCK cells, can undergo morpho- genesis and develop into polarized, elongated and branching tubule structures under the stimulation of hepatocyte growth factor (HGF) $[1,2]$. In our previous study, we successfully isolated several MDCK sub-clones, y224 and m634, that exhibited either cystic or tubular phenotypes in low concentration of collagen gel without HGF stimulation $[3,4]$. These cells became excellent models for studying novel regulatory mechanisms of branching tubulogenesis. 
It has been demonstrated that extracellular matrix proteins (ECM) are key regulators for branching morphogenesis. Fibronectin, for example, is synthesized by groups of adherent cells that assemble it into a fibrillar network $[5,6]$. Fibronectin is known to provide survival cues for MDCK cell morphogenesis and development of mouse submandibular salivary gland, kidney as well as lung [79]. Laminin, another example of ECM, is crucial in the end bud persistence and ductal elongation in the developing mammary gland [10]. Despite the importance of extracellular matrix proteins, integrins (the membrane receptors of ECM) are also of particular significance, as the potential of branching tubulogenesis in MDCK cells was inhibited by the decrease in $\alpha 3$ integrin expression [3]. Monoclonal antibodies against either $\alpha 6$ or $\beta 1$ subunit were shown to reduce kidney tubulogenesis in vitro [11], while temporal and spatial changes in integrin expression promoted branching morphogenesis of the developing collecting system in vivo [12].

Focal adhesion proteins are downstream of ECM and interact with integrins. Among all focal adhesion complex proteins, focal adhesion kinase (FAK) is of greatest significance. FAK connects both integrin and growth factor receptors with the intracellular signaling pathways and has five tyrosine phosphorylation sites (Y397, Y407, Y577, Y861, Y925), each mediating a different cellular function. In general, FAK-mediated signaling affects cell motility, cell survival, and cell cycle progression $[13,14]$. Because of the FAK involvement in the downstream cascade of integrin signaling, we wished to examine the role FAK in renal branching tubulogenesis.

In the present study we isolated several clones from MDCK cell line, i.e. cyst and mass clones, according to the methods described previously [3]. When both clones were cultured in $0.1 \%$ collagen gel, cyst clones formed cysts, while mass cells formed tubular structures. The investigation of the protein profiles showed that FAK expression was higher in mass clones then cyst clones. Overexpression of wild type FAK in cyst clones did not facilitate branching tube morphology when cultured in collagen gel. However, decrease of FAK expression levels by antisense cDNA in mass clones resulted in a marked reduction in branching tubule morphology in collagen gel. Our data suggest that FAK is required, but not sufficient for renal branching tubulogenesis.

\section{Materials and methods \\ Cell lines and transfection}

Cells were maintained in Dulbecco's modified Eagle's medium (DMEM, GIBCO BRL) supplemented with 5\% fetal bovine serum under $5 \% \mathrm{CO}_{2}$ at $37^{\circ} \mathrm{C}$. MDCK II $3 \mathrm{~B} 5$ cells overexpressing $\mathrm{HA}$ epitope-tagged wild type FAK, FRNK were the gifts from Dr. Hong-Chen Chen (National
Chung Hsing University, Taiwan). To generate cells stably expressing wild type FAK or antisense FAK cDNA, cells were grown on $60 \mathrm{~mm}$ dishes and transfected essentially using LipofectAMINE according to the manufacturer's protocol. Clones were selected in hygromycin-containing medium and screened by immunoblotting with anti-HA or anti-FAK.

\section{Materials}

Fetal bovine serum and LipofectAMINE were purchased from Life Technologies, Inc. G418 sulfate and hygromycin were purchased from Calbiochem (San Diego, CA). Recombinant human HGF were purchased from Sigma (St. Louis, MO).

\section{Preparation of collagen gel}

Type I collagen was prepared from rat tail tendons according to the established procedure [15]. The collagen stock solution (1\%), was composed of $1 \mathrm{~g}$ (dry weight) of rat tail tendons dissolved in $100 \mathrm{ml} 0.025 \mathrm{~N}$ acetic acid. For the preparation of $0.1 \%$ collagen gel, 1 vol of collagen stock was mixed with $5.7 \times$ DMEM $(1 \mathrm{vol}), 2.5 \% \mathrm{NaHCO}_{3}$ ( $0.5 \mathrm{vol}), 0.1 \mathrm{M}$ HEPES ( $1 \mathrm{vol}), 0.17 \mathrm{M} \mathrm{CaCl}_{2}(0.03 \mathrm{vol})$, $1 \mathrm{~N} \mathrm{NaOH}(0.03 \mathrm{vol})$, and $1 \times$ culture medium $(6.34 \mathrm{vol})$ under chilled conditions.

\section{Tubulogenesis}

For tubulogenesis assays, MDCK cells were suspended at a concentration of $2 \times 10^{4} \mathrm{cells} / \mathrm{ml}$ in cold collagen solution as described above. An aliquot $(1 \mathrm{ml})$ of cell suspension was dispensed into a well of 6-well plate and allowed to gel for about $20 \mathrm{~min}$ at $37^{\circ} \mathrm{C}$ before adding $1 \mathrm{ml}$ of medium containing $5 \%$ serum with or without HGF. The medium was changed daily. After 5 days, the tubules were photographed using a phase contrast microscope.

\section{Collection of cell lysate and Western blot}

The cells were cultured on dish for $24 \mathrm{hrs}$. Cell lysates were harvested in modified RIPA buffer $(150 \mathrm{mM} \mathrm{NaCl}, 1 \mathrm{mM}$ EGTA, 50 mM Tris pH 7.4, 10\% glycerol, $1 \%$ Triton X-100, $1 \%$ sodium deoxycholate, $0.1 \%$ SDS, and Complete ${ }^{\mathrm{TM}}$ ) and the lysates were analyzed by Western blot using antibodies against FAK, fibronectin, vinculin, talin, paxillin and Src (Transduction Lab), phosphorylated Y397, Y407, Y577, Y861, Y925 (Biosource), $\alpha 2, \alpha 3, \beta 1$ integrin (Chemicon), HA (Roche), p130cas, $\beta$-actin (Sigma) and visualized using enhanced chemiluminescence (ECL) system (Amersham-Pharmacia).

\section{Migration assay}

Cells were collected by trypsinization and suspended in serum-free medium at $5 \times 10^{5} \mathrm{cells} / \mathrm{ml}$. Migration assays were carried out in a Neuro Probe 48-well chemotaxis chamber (Cabin John, MD). The medium containing collagen $(10 \mu \mathrm{g} / \mathrm{ml})$ was added to the lower chamber. The 
lower and upper chambers were separated by a polycarbonate membrane $(8 \mu \mathrm{m}$ pore size, Poretics, Livermore, CA). Cells were allowed to migrate for $6 \mathrm{hrs}$ at $37^{\circ} \mathrm{C}$ in a humidified atmosphere containing $5 \% \mathrm{CO}_{2}$. The membrane was fixed in methanol for $10 \mathrm{~min}$ and stained with modified Giemsa stain (Sigma) for $1 \mathrm{hr}$. Cells on the upper side of the membrane were removed by cotton swabs. Cells on the lower side of the membrane were counted using a light microscope at $200 \times$ magnification.

\section{Plasmid construction}

Total RNA was isolated from cultured MDCK cells by the standard guanidium isothiocyanate extraction method. Reverse transcription (RT) and polymerase chain reaction (PCR) were performed. Primers used to amplify a $712 \mathrm{bp}$ segment of canine FAK cDNA were as follows: forward primer 5'-TACCTACCGAGGTCTCAGTTAG-3' (nucleotides 1305 to 1326 in human FAK CDNA) and reverse primer 5'-GCGCTGATCTTCTTCCATTTCC-3' (nucleotides 1995 to 2016 in human FAK cDNA). This segment is conserved in human, rat, mouse, and chicken FAK. The 712 bp PCR product was further mixed with XhoI cutting sequence, purified from $1 \%$ agarose, cleaved with XhoI endonuclease, and ligated into pMSCVhyg to create pMSCVFAKhyg, which was finally transfected into E. coli. The canine FAK sequence is approximately $89 \%$ homologous with the human FAK cDNA.

\section{Fibronectin assembly assay}

The method to determine the fibronectin matrix assembly [16] was modified. To detect the amount of assembled fibronectin in cells cultured on dish, MDCK cells were cultured in the culture medium for 24 hrs then rinsed twice with ice cold PBS. Cells were lysed in DOC lysis buffer ( $2 \%$ sodium deoxycholate, $20 \mathrm{mM}$ Tris-Cl, $\mathrm{pH} 8.8,2 \mathrm{mM}$ phenylmethysulfonyl fluoride, $2 \mathrm{mM}$ EDTA, $2 \mathrm{mM}$ iodoacetic acid, and $2 \mathrm{mM} \mathrm{N}$-ethylmaleimide) and cell lysate was harvested using a cell scraper (Nunc). To detect the amount of assembled fibronectin MDCK cells were cultured in $0.1 \%$ collagen gel for 6 days. Collagen gel was digested by $0.2 \%$ collagenase under $37^{\circ} \mathrm{C}$ for $10 \mathrm{~min}$. Cells were collected by centrifugation at $1200 \mathrm{rpm}$ and then lysed in the DOC lysis buffer. Lysates were centrifuge for $15 \mathrm{~min}$ at $13,000 \mathrm{rpm}$ in $4^{\circ} \mathrm{C}$ to separate DOC-insoluble matrix. The DOC-insoluble fibronectin was then dissolved in SDS-containing buffer (1\% SDS, $20 \mathrm{mM}$ Tris-Cl, pH 8.8, 2 mM PMSF, 2 mM EDTA, 2 mM iodoacetic acid, $2 \mathrm{mM}$ N-ethylmaleimide) and analyzed by Western blot to detect fibronectin.

\section{Results}

MDCK cells form two different phenotypes when cultured in $0.3 \%$ type I collagen gel. Most of these cells develop into polarized cysts with an obvious central cavity, while the others form cell aggregates [3]. In the present study, we successfully isolated MDCK subclones that consistently developed either cysts (cyst clones - CA4, CA6) or cell aggregates (mass clones - M610, M611, M612) in $0.3 \%$ collagen gel for at least five passages. When these clones were cultured in $0.1 \%$ collagen gel for 6 days, cyst clones continued to display cystic morphogenesis, whereas mass clones developed into tubular structures (Fig. 1).

Although cyst clones and mass clones exhibited different morphogenesis in $0.1 \%$ collagen gel, they showed similar growth capability (Fig. 2a). A notable difference in the morphology between cyst clones and mass clones was observed when the cells were cultured on the culture dish. Cyst clones revealed a well-developed association between neighboring cells and formed aggregated islands. In contrast, mass clones showed more scattered morphology when cultured on the dish (Fig. 2b). The scattered morphology indicates that mass clones might have greater motility than cyst clones. To test this hypothesis, we used Boyden chamber transwell assay to analyze the migration ability of these two clones. Under collagen stimulation, mass clones showed higher migration ability than cyst clones (Fig. 2c).

Since migration ability is highly regulated by focal adhesion related proteins, we reasoned that the expression of these proteins may be involved in regulating cell morphogenesis. We analyzed some focal adhesion related proteins including extracellular matrix (fibronectin), receptors for extracellular matrix ( $\alpha 2, \alpha 3$ and $\beta 1$ integrins) and focal adhesion complex proteins (FAK, p130cas, talin, vinculin, paxillin, Src). Among these proteins, none of them showed apparent differences between these two clones except for the expression levels of FAK, which was markedly higher in mass than cyst clones (Fig. 3a). We furthered detected the phosphorylation level of different tyrosine phosphorylation sites on FAK in CA6 and M610 cells. As shown in Fig. 3b, M610 cells showed higher phosphorylation level in all FAK tyrosine phosphorylation sites compared to CA6 cells. These data suggest that FAK may be involved in regulating tubule formation.

We further investigated whether FAK expression and phosphorylation level is higher in mass than in cyst clones when cultured in $0.1 \%$ collagen gel. Cyst and mass clones were cultured in $0.1 \%$ collagen gel for 6 days and FAK expression and phosphorylation levels were examined. When cells were cultured in $0.1 \%$ collagen gel, mass clones showed consistently higher FAK levels than cyst clones within 6 days (Fig. 4a). Furthermore, M610 cells showed higher phosphorylation levels in all FAK tyrosine phosphorylation sites compared to CA6 cells when cultured in $0.1 \%$ collagen gel for 6 days (Fig. $4 \mathrm{~b}$ ). 

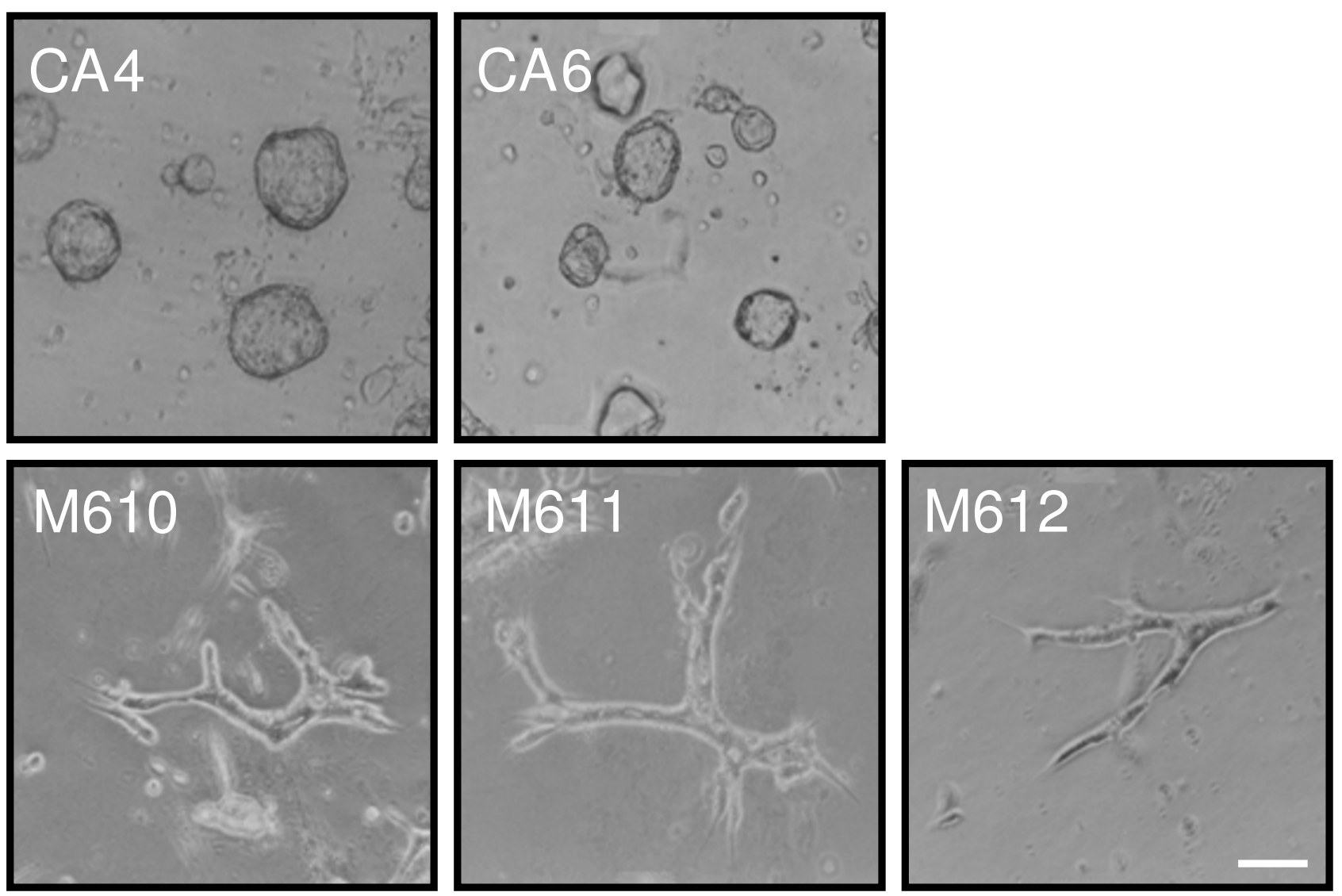

Figure I

Morphogenesis of cyst and mass clones cultured in $\mathbf{0 . 1} \%$ type I collagen gel. Cyst clones (CA4, CA6) and mass clones (M6I0, M6II, M6I2) were cultured in 0.1\% collagen gel for 6 days. Images were taken under a light microscope. Bar $=100 \mu \mathrm{m}$.

In order to define the role of FAK in regulating tubule formation, we established several FAK transfectants in cyst and mass clones. To elevate the FAK expression level, wild type FAK was overexpressed in CA6 cells. In addition, antisense cDNA was used to decrease the FAK expression level in M610 cells. Elevation of FAK in CA6 cells increased FAK Y397 phosphorylation level. Meanwhile, reduction of FAK expression in M610 cells also diminished the FAK Y397 phosphorylation level (Fig. 5a). We further observed the morphology of those FAK transfectants when cultured on dish. FAK-overexpressed CA6 cells (CF5) formed aggregated islands similar to CA6 and control cells (CV). However, when antisense FAK-overexpressed M610 cells (MF9, MF-13) were cultured on dish, they showed markedly reduced scattered morphology and elevated cell-to-cell association compared to M610 and control cells (MV) (Fig. 5b). To measure whether cell motility was altered by different FAK expression, migration capability of different FAK transfectants was analyzed by Boyden chamber with collagen as a chemoattractant. Increasing the expression level of FAK in CA6 cells did not elevate migration ability significantly; however decreasing the expression level of FAK in M610 cells reduced the migration ability in a dosedependent manner (Fig. 5c).

To determine whether FAK was involved in regulating tubule formation, all the FAK transfectants were cultured in $0.1 \%$ collagen gel for 6 days. FAK-overexpressed CA6 cells (CF5) continued to form cysts. The number and size of cysts were similar in CA6 cells and FAK-overexpressed CA6 cells (data not shown). In contrast, antisense FAKoverexpressed M610 cells (MF-9, MF-13) did not develop into tube-like structures, but instead formed small cell masses. In addition, decreasing the expression level of FAK in M610 cells reduced the tubule formation in a dosedependent fashion (Fig. 6a). We next investigated whether FAK phosphorylation level was linked to tubule formation. FAK transfectants were cultured in $0.1 \%$ collagen gel for 6 days and FAK phosphorylation levels were examined. As shown in Fig. 6b, overexpression of FAK in CA6 cells did not augment FAKY397 phosphorylation. However, a decrease of FAK in M610 cells reduced FAKY397 
A

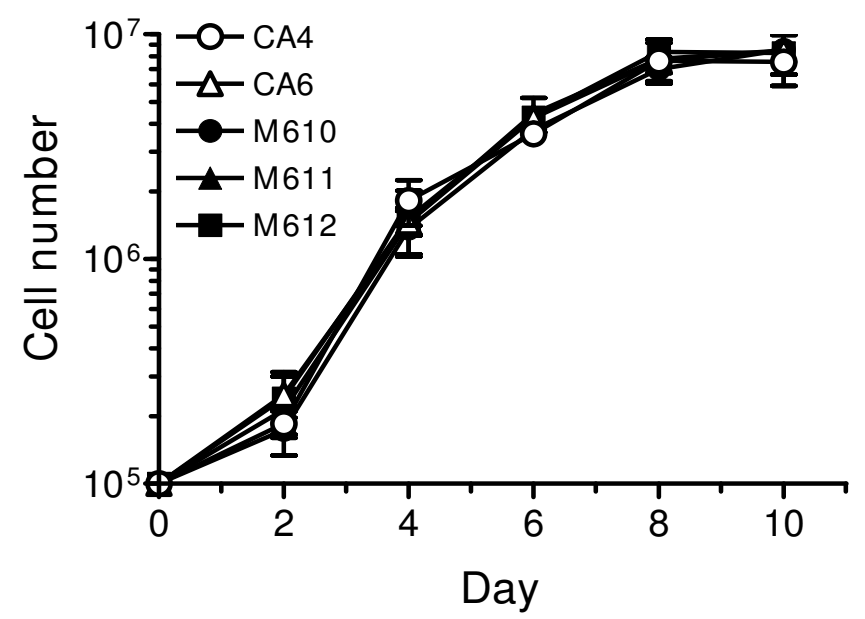

B
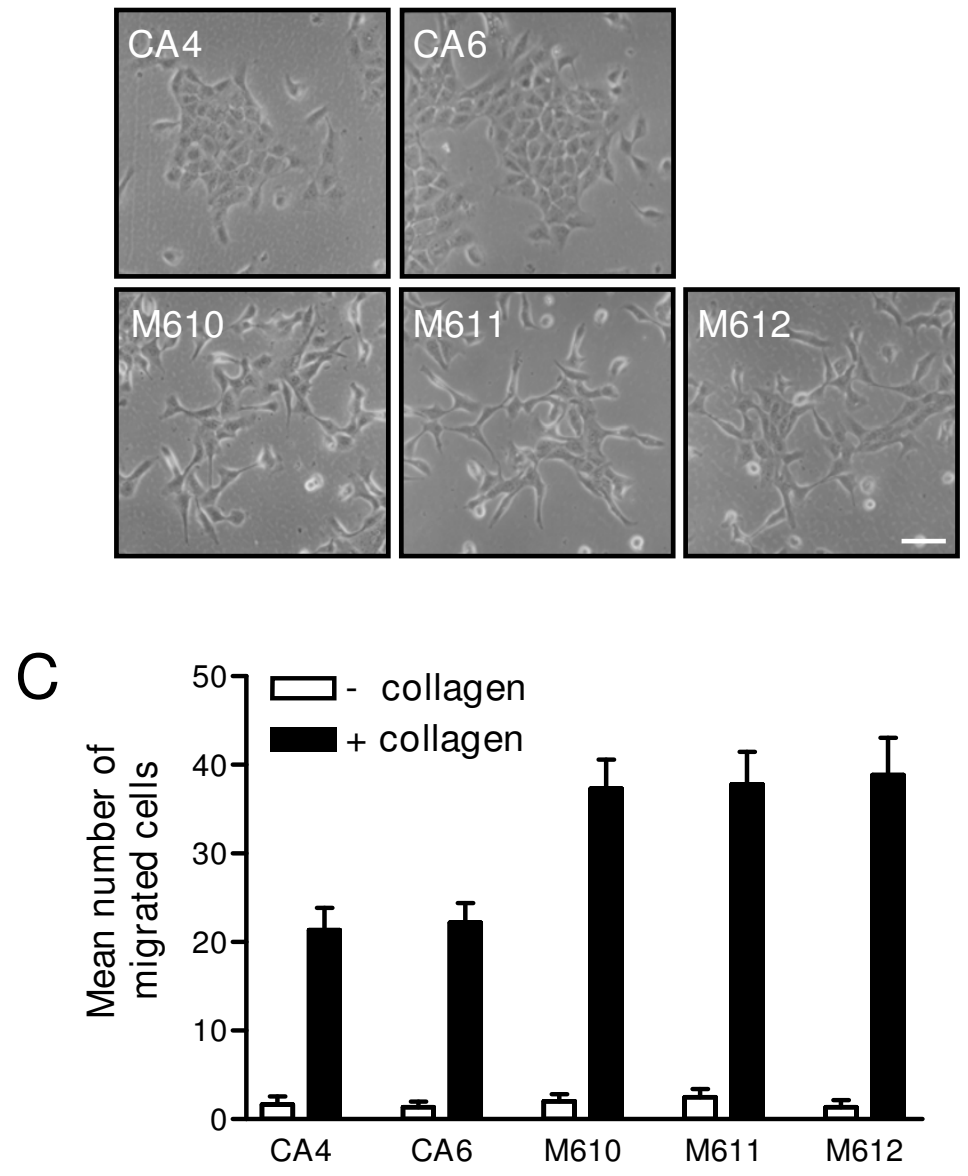

Figure 2

Characteristics of cyst and mass clones cultured on dish. (a) Morphology of cyst clones (CA4, CA6) and mass clones (M6I0, M6II, M6I2) cultured on dish for 24 hrs. Images were taken under a light microscope. Bar $=30 \mu \mathrm{m}$. (b) Growth curve of cyst and mass clones cultured on dish for 10 days. (c) Cyst clones and mass clones subjected to migration assays using collagen as a chemoattractant. Migrated cells were fixed, stained, and counted under a light microscope. Values (mean \pm S.E.) are from 15 fields and 3 independent experiments. 


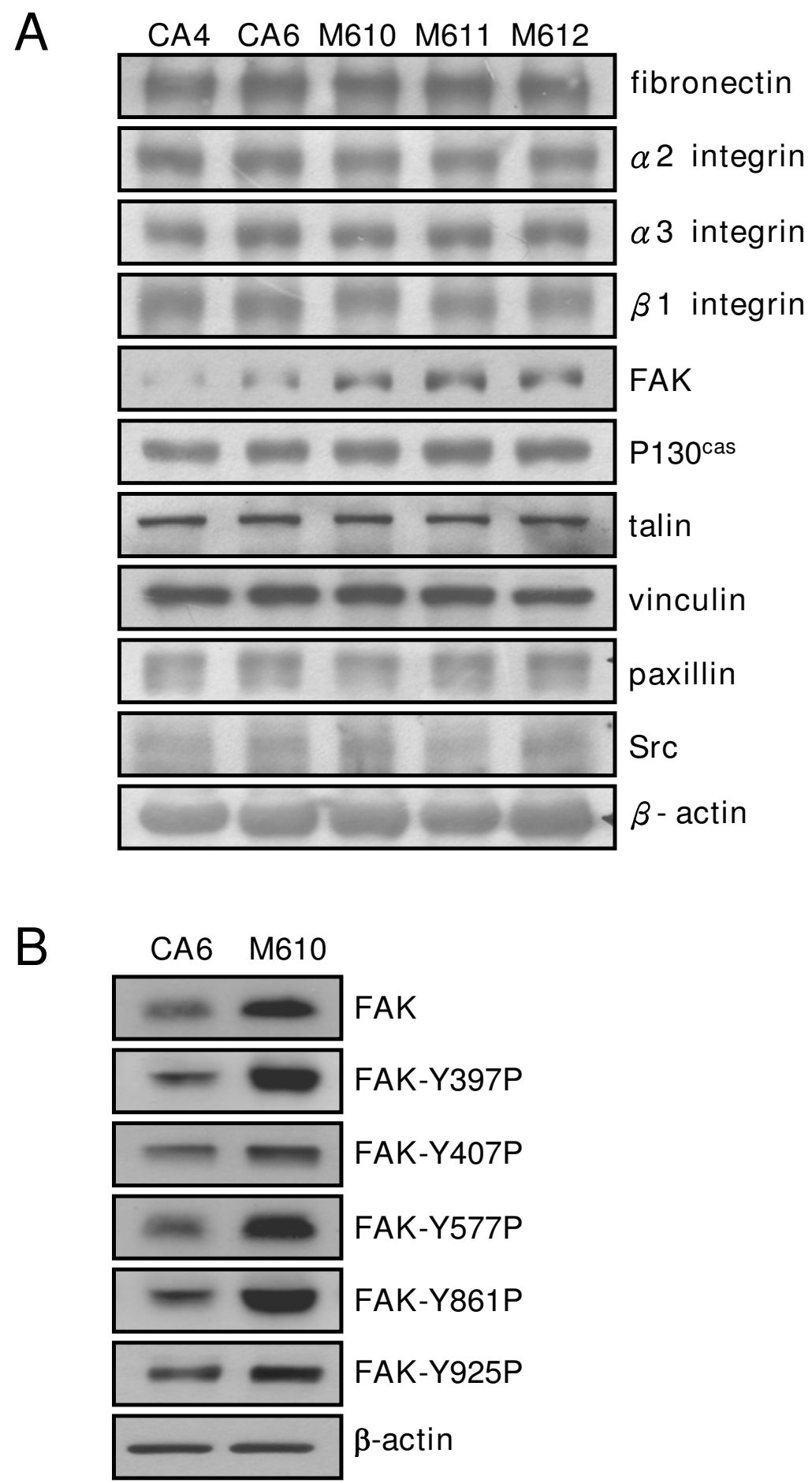

Figure 3

Focal adhesion proteins expression in cyst and mass clones. (a) Cyst clones (CA4, CA6) and mass clones (M6I0, M6I I, M6I2) were cultured on dish for $24 \mathrm{hrs}$. Lysates were analyzed by Western blot using antibodies to detect fibronectin, $\alpha 2, \alpha 3, \beta I$ integrin, FAK, PI30cas, talin, vinculin, paxillin, Src, and $\beta$-actin. (b) CA6 and M6I0 cells were cultured on dish for 24 hrs. Lysates were analyzed by Western blot using antibodies to detect FAK and Y397, Y406, Y577, Y86I, Y925 phosphorylated FAK levels. 


\section{A}
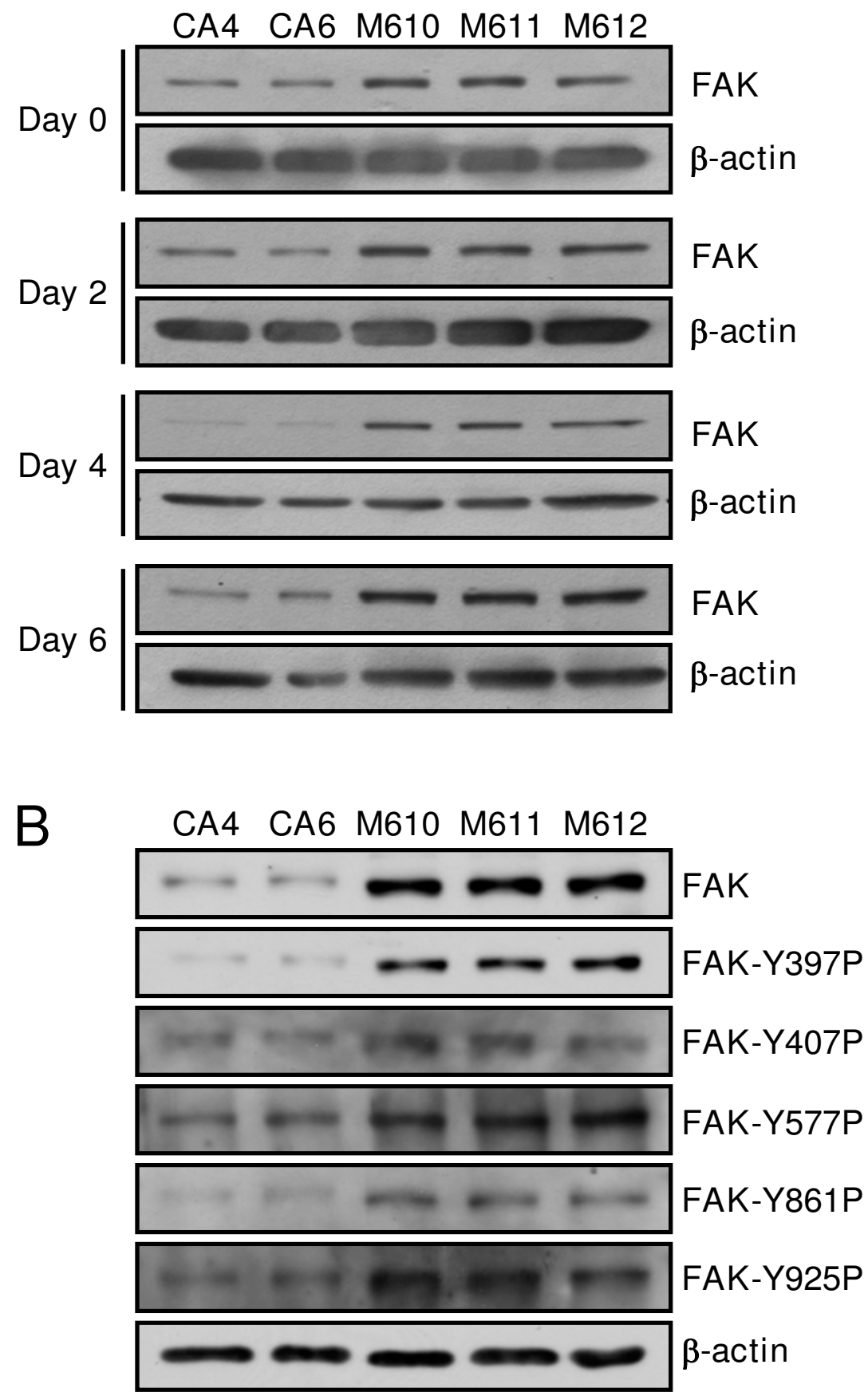

\section{Figure 4}

Comparison of FAK and FAK phosphorylation levels between cyst and mass clones cultured in $0.1 \%$ collagen gel. (a) Cyst clones (CA4, CA6) and mass clones (M6I0, M6II, M6I2) were cultured in $0.1 \%$ collagen gel for indicated time. Lysates were analyzed by Western blot using antibodies to detect FAK and $\beta$-actin. (b) Cyst clones (CA4, CA6) and mass clones (M6IO, M6II, M6I2) were cultured in $0.1 \%$ collagen gel for 6 days. Lysates were analyzed by Western blot using antibodies to detect FAK and Y397, Y406, Y577, Y86I, Y925 phosphorylated FAK. 
A CA6 CV CF5 M610 MV MF-9 MF-13

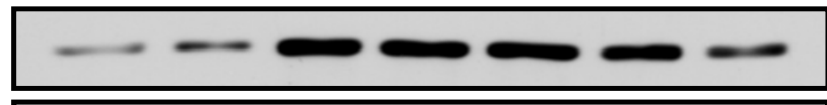

FAKY397P

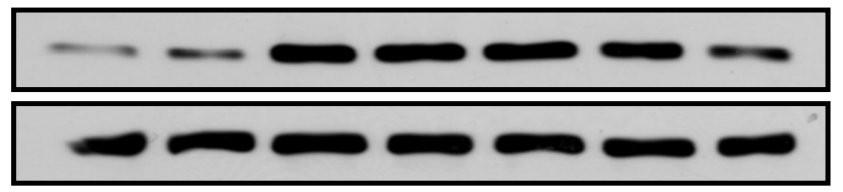

FAK

$\beta$-actin

B
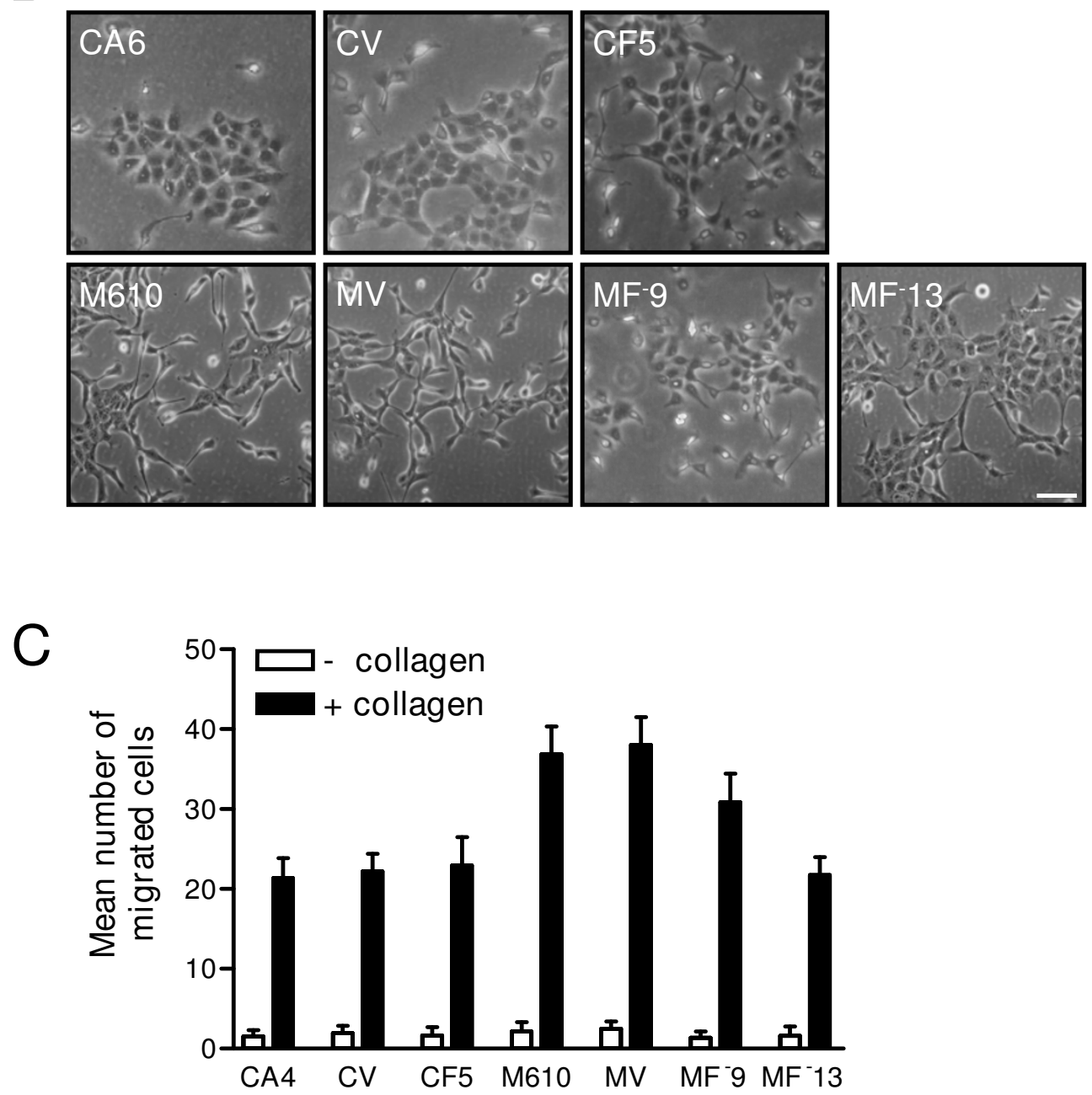

\section{Figure 5}

Morphology and migration ability of FAK transfectants in cyst and mass clones. (a-b) CA6, M6I0, control clones (CV, MV), wild type FAK overexpressed-CA6 cell (CF5), or antisense FAK overexpressed-M6I0 cells (MF-9, MF-I3) were cultured on dish for $24 \mathrm{hrs}$. (a) Lysates were analyzed by Western blot using antibodies to detect FAK and $\beta$-actin. (b) Cell morphology; images were taken under a light microscope. Bar $=30 \mu \mathrm{m}$. (c) Cells were subjected to migration assays using collagen as a chemoattractant. Migrated cells were fixed, stained, and counted under a light microscope. Values (mean \pm S.E.) are from I 5 fields and 3 independent experiments. 
A
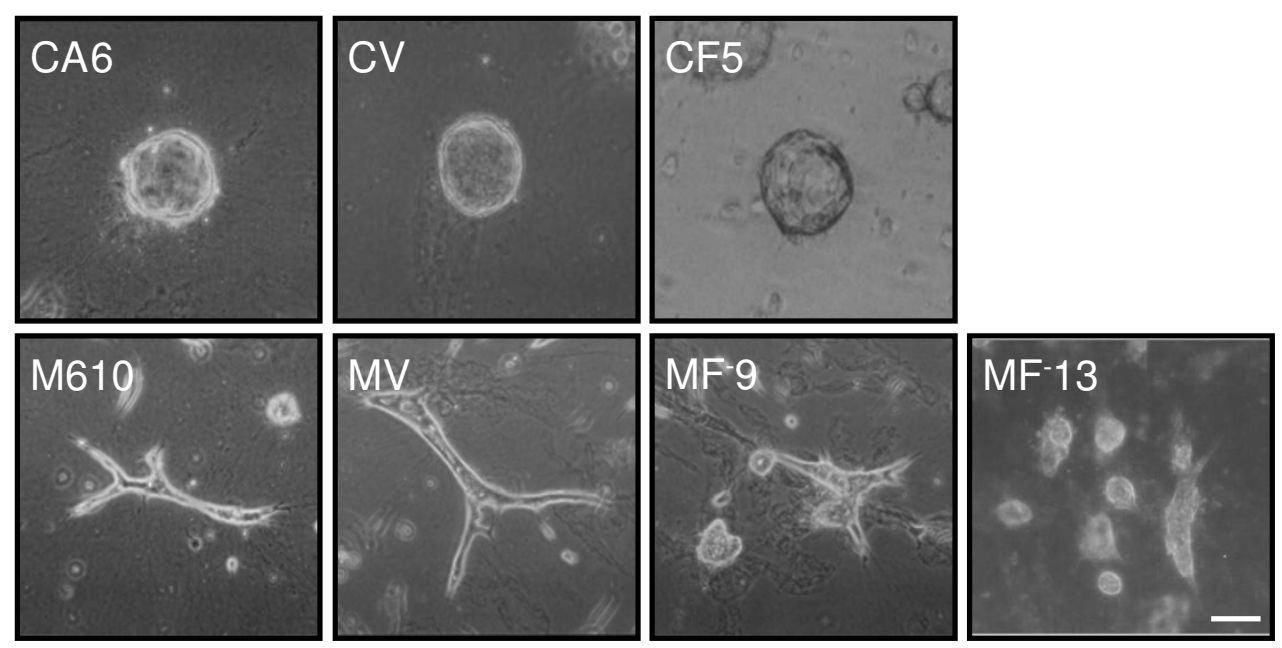

B
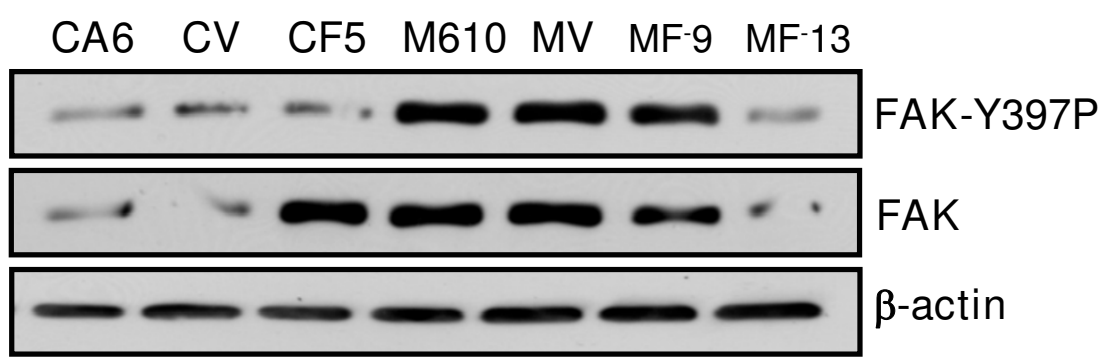

C

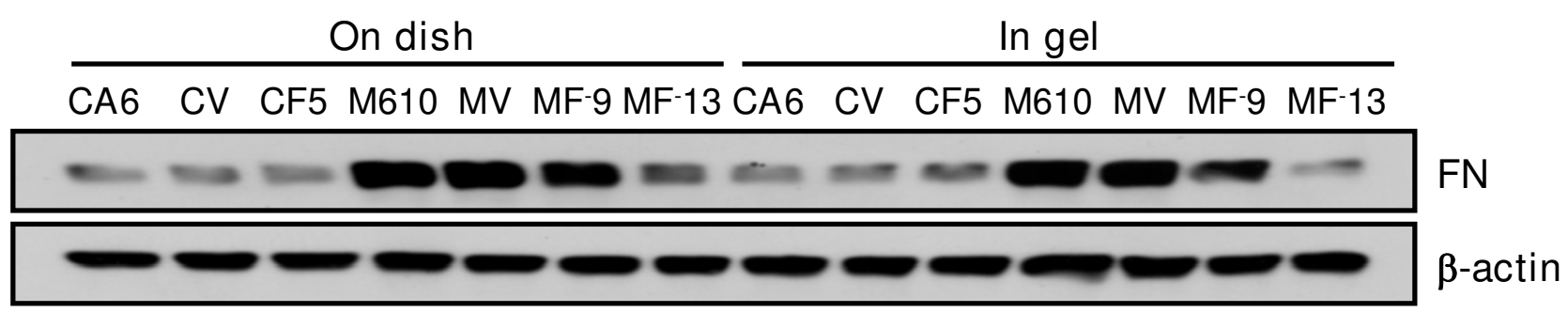

\section{Figure 6}

Characteristics of FAK transfectants in cyst and mass clones cultured in $\mathbf{0 . 1 \%}$ collagen gel. (a-b) CA6, M6I0, control clones (CV, MV), wild type FAK overexpressed-CA6 cell (CF5), or antisense FAK overexpressed-M6IO cells (MF-9, MF-13) were cultured in $0.1 \%$ collagen gel for 6 days. (a) Morphology of cells, images were taken under a light microscope. Bar $=100$ $\mu \mathrm{m}$. (b) Lysates were analyzed by Western blot using antibodies to detect FAK, Y397 phosphorylated FAK and $\beta$-actin. (c) CA6, M6I0, control clones (CV, MV), wild type FAK overexpressed-CA6 cell (CF5), or antisense FAK overexpressed-M610 cells (MF-9, MF-13) were cultured on dish for 24 hrs or in $0.1 \%$ collagen gel for 6 days. Assembled fibronectin was extracted and analyzed by Western blot using antibodies to detect fibronectin. 
phosphorylation. Taken together, our data indicate that FAK is required, but not sufficient for tubulogenesis.

Our previous study demonstrated that deposition of extracellular fibronectin is crucial for tubulogenesis [8]. To identify whether FAK-overexpressed CA6 cells fail to form tubules due to an inadequate fibronectin assembly, we analyzed the potential of fibronectin assembly in FAK transfectants cultured on dish for 24 hrs or in $0.1 \%$ collagen gel for 6 days. The results showed that overexpression of FAK in CA6 cells did not affect the amount of assembled fibronectin. In contrast, a decrease of FAK in M610 cells was accompanied by a reduction of fibronectin assembly (Fig. 6c). Thus, a decrease of FAK in M610 cells leads to down-regulation of tubule formation, which cannot be attributed to the reduction of fibronectin assembly. On the other hand, overexpression of wild type FAK in CA6 cells did not trigger tubulogenesis, which may be due to insufficient fibronectin assembly.

To further investigate whether FAK is also involved in HGF-dependent tubulogenesis, 3B5 cells, a subclone of MDCK cells, harboring wild type FAK or dominant negative FRNK (FAK-related non-kinase) were employed. Cells were cultured in $0.3 \%$ collagen gel with different dosage regimen of HGF stimulation. FAK-overexpressed cells were more sensitive to HGF stimulation in forming branching tubules, whereas FRNK-overexpressed cells did not survive (Fig. 7). Taken together, our data show that FAK plays an important role in regulating branching tubulogenesis in both HGF-dependent and HGF-independent models.

\section{Discussion}

In this study, we provide evidence that FAK is a key element in renal tubulogenesis. First, we isolated two different clones from MDCK cells, which proved to be a successful, HGF-independent model for tubule morphogenesis. We showed that mass clones forming tubule structures exhibited higher expression of FAK protein compared to cyst clones forming cystic morphogenesis in $0.1 \%$ collagen gel. Furthermore, the elevated FAK expression level in cyst clones cannot promote tubule formation. However, a decrease of FAK expression level in mass clones dramatically reduced branching tubule formation. Finally, we also demonstrated that FAK facilitates HGFinduced branching tubulogenesis since cells harboring wild type FAK sensitized the dose of HGF stimulation. These results indicate that FAK is involved in the regulation of both the HGF-dependent and HGF-independent branching morphogenesis.

The effects of FAK in kidney development in vivo have not been studied before, because knockout FAK in mice leads to embryonic lethal at day 8.5 [17]. However, observa- tions from FAK-/- mice showed that FAK is an important regulator in vascular morphogenesis since FAK-deficient endothelial cell failed to organize into vascular networks $[18,19]$. Indeed, FAK has been shown to be required in blood vessel morphogenesis [20]. Furthermore, conditional FAK knockouts in endothelial cells led to defective angiogenesis [21]. Therefore, it is likely that conditional FAK knockouts in early ureteric bud may result in the failure of renal tubulogenesis in vivo.

Branching morphogenesis is a multi-step process that requires sequential cell adhesion to extracellular matrix, cell spreading, cell proliferation, and cell migration to ultimately form multicellular tube-like structures [22]. In the current study, we showed that decrease of FAK expression in M610 cells inhibited branching morphogenesis that was accompanied by the reduction of FAK phosphorylation and cell motility. However, overexpression of FAK in CA6 cells failed to elevate migration capacity, tubule formation and phosphorylation of FAK Y397 in collagen gel. Since there was a high agreement between the phosphorylation level of FAK Y397 and tubule formation, it is likely that FAK 397 phosphorylation may also play an important role in renal morphogenesis in MDCK cells.

Our previous study showed that MDCK cells cultured in collagen gel exhibited deposition of fibronectin underlying the epithelium, which may prevent apoptosis and therefore can possibly facilitate cyst or tubule growth of MDCK cells [7,8]. Recently, FAK was reported to promote organization of fibronectin matrix in endothelial cells [23]. Likewise, FAK may also regulate fibronectin assembly in MDCK cells. We reason that the decrease of FAK expression in M610 cells results in poor fibronectin deposition and hence leads to inhibition of branching tubulogenesis. Moreover, it has been reported that FAKY397 phosphorylation is highly regulated by substratum stiffness [24]. Well-assembled fibronectin around the cell may serve as a stiff substrate to further promote FAKY397 phosphorylation. Thus, mass clones are likely to possess higher potential to assemble fibronectin and to induce greater FAK phosphorylation. Finally, there may be a positive feedback loop in regulating FAK phosphorylation and fibronectin assembly during renal tubulogenesis.

To summarize, we have shown that FAK is critical for renal branching tubulogenesis through regulation of cell migration and fibronectin assembly. Our studies revealed FAK is involved in renal tubulogenesis, which may be also involved in other tissue morphogenesis such as mammary gland, pancreas or lung.

\section{Competing interests}

The authors declare that they have no competing interests. 


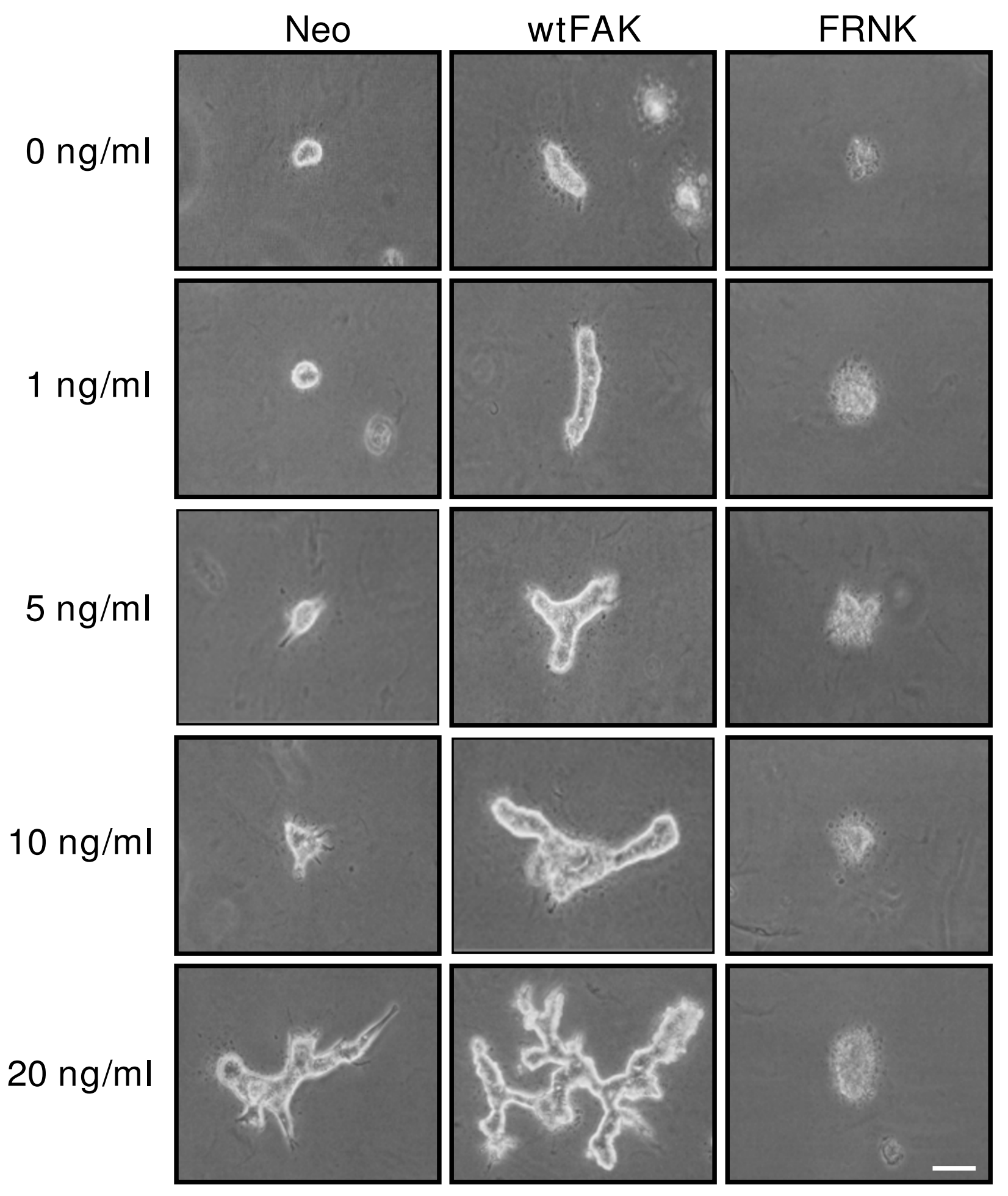

Figure 7

The effect of FAK on tubulogenesis in $\mathbf{0 . 3} \%$ collagen gel with different dosage of hepatocyte growth factor. Morphology of 3B5 cells stably transfected with FAK, FRNK, and Neo were cultured in $0.3 \%$ collagen gel in the presence of HGF of $0, \mathrm{I}, 5,10,20 \mathrm{ng} / \mathrm{ml}$, respectively, for 7 days. Bar $=100 \mu \mathrm{m}$. 


\section{Authors' contributions}

WCW participated in the design and performed statistical analysis of the results and drafted the manuscript. AKK carried out part of the experimentation. MJT conceived of the study and participated in its design and coordination of the study. All authors read and approved the final manuscript.

\section{Acknowledgements}

This work was supported by National Health Research Institute grant NHRI-EX95-9430SI and the National Science Council grant 95-2 I20-M006-007 to Ming-Jer Tang

\section{References}

I. Montesano R, Schaller G, Oric L: Induction of epithelial tubular morphogenesis in vitro by fibroblast-derived soluble factors. Cell |99I, 66:697-7II.

2. Montesano R, Matsumoto K, Ribeiro E, Nigam SK: Epithelial tubulogenesis through branching morphogenesis: Relevance to collecting system development. J Am Soc Nephrol 1995, 6:1151-1159.

3. Jiang ST, Liu HS, Chen HC, Chuang WJ, Tang MJ: The role of $\alpha 3 \beta$ I integrin in tubulogenesis of MDCK cells. Kidney Int 200I, 59:1770-8.

4. Lin HH, Yang TP, Jiang ST, Liu HS, Tang MJ: Inducible expression of bcl-2 by the lac operator/repressor system in MDCK cells. Am J Physiol 1997, 273(2 pt 2):300-306.

5. Mao Y, Schwarzbauer JE: Fibronectin fibrillogenesis, a cell-mediated matrix assembly process. Matrix Biol 2005, 24:389-399.

6. Wierzbicka-Patynowski I, Schwarzbauer JE: The ins and outs of fibronectin matrix assembly. J Cell Sci 2003, I I 6:3269-3276.

7. Jiang ST, Chiang HC, Cheng MS, Yang TP, Chuang WJ, Tang MJ: The role of fibronectin deposition in cystogenesis of MDCK cells. Kidney Int 1999, 56:92-103.

8. Jiang ST, Yang TP, Chuang WJ, Tang MJ: The role of fibronectin deposition in branching morphogenesis of MDCK cells. Kidney Int 2000, 57:1860-1867.

9. Sakal T, Larsen M, Yamada KM: Fibronectin requirement in branching tubulogenesis. Nature 2003, 423:876-88I.

10. Klinowska TC, Soriano JV, Edwards GM, Oliver JM, Valentijn AJ, Montesano $\mathrm{R}$, Streuli $\mathrm{CH}$ : Laminin and $\beta$ I integrins are crucial for normal mammary gland development in the mouse. Dev Biol 1999, 21 5:13-32.

II. Falk M, Salmivirta K, Durbeej M, Larsson E, Ekblom M, Vestweber D, Ekblom P: Integrin $\alpha 6 \beta I$ is involved in kidney tubulogenesis in vitro. J Cell Sci 1996, 109:280I-2810.

12. Chen D, Roberts R, Pohl M, Nigam S, Kreidberg J, Wang Z, Heino J, Ivaska J, Coffa S, Harris RC, Pozzi A, Zent R: Differential expression of collagen- and laminin-binding integrins mediates ureteric bud and inner medullary collecting duct cell tubulogenesis. Am J Physiol Renal Physiol 2004, 287:602-6II.

13. Parsons JT: Focal adhesion kinase: the first ten years. J Cell Sci 2003, II 6: | 409-1416.

14. Schlaepfer DD, Mitra SK: Multiple connections link FAK to cell motility and invasion. Curr Opin Genet Dev 2004, I 4:92-I0I.

15. Wang YK, Wang YH, Wang CZ, Sung JM, Chiu WT, Lin SH, Chang $\mathrm{YH}$, Tang MJ: Rigidity of collagen fibrils controls collagen gelinduced down-regulation of focal adhesion complex proteins mediated by $\alpha 2 \beta$ I integrin. J Biol Chem 2003, 278:21886-21892.

16. Wierzbicka-Patynowski I, Mao Y, Schwarzbauer JE: Analysis of fibronectin matrix assembly. Curr Protoc Cell Biol 2004, Chapter I0(unit I0): 12 .

17. Ilic $D$, Kanazawa S, Furuta $Y$, Yamamoto T, Aizawa S: Impairment of mobility in endodermal cells by FAK deficiency. Exp Cell Res 1996, 222:298-303.

18. Furuta $Y$, llic D, Kanazawa S, Takeda N, Yamamoto T, Aizawa S: Mesodermal defect in late phase of gastrulation by a targeted mutation of focal adhesion kinase, FAK. Oncogene 1995, I I:1989-1995.

19. Ilic D, Furuta $Y$, Kanazawa S, Takeda N, Sobue K, Nakatsuji N, Nomura S, Fujimoto J, Okada M, Yamamoto T, Aizawa S: Reduced cell motility and enhanced focal adhesion contact formation in cells from FAK deficient mice. Nature 1995, 377:539-544.

20. Ilic D, Kovacic B, McDonagh S, Jin F, Baumbusch C, Gardner DG, Damsky CH: Focal adhesion kinase is required for blood vessel morphogenesis. Circ Res 2003, 92:300-307.

21. Shen TL, Park AY, Alcaraz A, Peng X, Hang I, Koni P, Flavell RA, Gu $\mathrm{H}$, Guan JL: Conditional knockout of focal adhesion kinase in endothelial cells reveals its role in angiogenesis and vascular development in late embryogenesis. J Cell Biol 2005, 169:941-952.

22. O'Brien LE, Zegers MM, Mostov KE: Opinion: building epithelial architecture: insights from three-dimensional culture models. Nat Rev Mol Cell Biol 2002, 3:53I-537.

23. Ilic D, Kanazawa S, Furuta Y, Yamamoto T, Aizawa S: Impairment of mobility in endodermal cells by FAK deficiency. Exp Cell Res 1996, 222:298-303.

24. Cukierman E, Pankov R, Stevens DR, Yamada KM: Taking cellmatrix adhesions to the third dimension. Science 200I, 294: $1708-1712$
Publish with BioMed Central and every scientist can read your work free of charge

"BioMed Central will be the most significant development for disseminating the results of biomedical research in our lifetime. "

Sir Paul Nurse, Cancer Research UK

Your research papers will be:

- available free of charge to the entire biomedical community

- peer reviewed and published immediately upon acceptance

- cited in PubMed and archived on PubMed Central

- yours - you keep the copyright 